\title{
Mejora de los programas de participación del personal
}

\author{
BERNARDO MARTÍNEZ MUT \\ Universidad de Valencia
}

\begin{abstract}
SUMMARY.-The involvement of the groups and the organizations members in quality improvement of the products and services that those manufacture and delivery, is a undeniable reality.

Specific projects have been carried out, inside of the more broad improvement programmes, for encouraching the employees involvement in the enterprises, two of which are described and analyzed in this article: Quality Circles and Suggestions System. Quality Circles are a group way for achieving that objective of involvement in the own work improvement, while the Suggestions are one of individual type and may be anonimous.

We analyze the opinions and the attitudes of the members of eight enterprises from «Comunidad Valenciana» to their participation in these programmes and the difficulties for carrying out the proposals. For gathering these data we are carrying out the proposals. For gathering these data we are applied a lateral thinking technique, so-called Metaplan.

Finally, according to the proposals of all the participants, we subscribe a range of measures for improving as the design as the execution of the programmes.
\end{abstract}

\section{INTRODUCCIÓN}

La participación de los miembros de los grupos y de las organizaciones en la mejora de la calidad de los productos y de los servicios que éstas fabrican o prestan es una realidad indiscutible. Pese a los cambios en los paradigmas explicativos de las razones o motivos que impulsan a los individuos a implicarse en la realización de su trabajo, los directivos, líderes y gestores de personal han considerado siempre necesario despertar el interés de las personas que colaboran con ellos para conseguir de este modo mejores resultados en los aspectos individual, grupal y organizativo de su trabajo.

McGregor ${ }^{1}$ formuló dos teorías que se apoyan en otras tantas concepciones diferentes sobre los individuos y los incentivos que se necesitan para estimularles: la teoría $\mathrm{X}$, típica representación de las tesis tayloristas en el diseño de las organizaciones y los puestos de trabajo, defendía que el trabajador necesita estímulos y presiones externas para moverse hacia unos objetivos impuestos $\mathrm{y}$, si no lo hace, debe ser presionado y amenazado por sus mandos o supervisores, puesto que considera es incapaz de encontrar fuentes internas de motivación; la teoría Y, por su parte, arranca de un concepto más 
positivo y optimista de las relaciones entre la empresa y el individuo que en ella trabaja, y cree, por ello, que la persona puede implicarse voluntariamente en la ejecución y mejora de sus tareas laborales si encuentra en los grupos y en las organizaciones ocasión de satisfacer sus necesidades y expectativas, que no tienen por qué ser contrapuestas ni contradictorias con las de la organización. Argyris, ${ }^{2}$, al definir su modelo de organización, considera que una cierta tensión entre el individuo y la organización lleva a los individuos a superarse y a crear empresas excelentes, por lo que se situaría cerca de los postulados de la teoría Y.

Tanto la teoría X como la Y defienden que son los factores preferentemente de carácter individual los que explican la implicación o compromiso de los individuos en la ejecución correcta y esperada de sus tareas laborales. La teoría Z, en cambio, enfatiza la dimensión comunitaria ${ }^{3}$ y analiza los factores, algunos de tipo cultural incluso, que provocan que las personas implicadas en su trabajo construyan unas organizaciones claramente diferenciadas, dentro de las cuales los valores y las creencias compartidas y una fuerte idea de compromiso con el proyecto empresarial son aspectos relevantes para explicar el logro y el mantenimiento de la participación del personal.

Podemos decir, en resumen, que todas las teorías -también las norteamericanas excelentes y las francesas del tercer tipo, por aludir a modelos cercanos en el espacio y en el tiempo ${ }^{4}$ - dan por supuesto que sin la participación del personal es inviable el logro de los objetivos deseados por los grupos y por las organizaciones, aunque cada una enfatice unos factores en lugar de otros para explicar que sea posible esta implicación o compromiso.

El origen de este artículo es una comunicación presentada como adenda a la ponencia sobre «Investigación-acción» ${ }^{5}$, en el XI Seminario Interuniversitario de Teoría de la Educación y tiene como objetivo «aportar unas conclusiones para la mejora de los programas de participación del personal, entendidos, aunque no de forma exhaustiva, como modalidades de intervención o práctica socio-educativa», las cuales conseguirán una mejora de la operatividad de estas estrategias o modalidades y, como consecuencia, una transformación positiva de la realidad social a la que se aplican, es decir, los grupos y los individuos en el seno de las organizaciones.

\section{LA PARTICIPACIÓN DEL PERSONAL ES UN VALOR EN ALZA EN LAS EMPRESAS: LOS CÍR- CUlos de CAlidad y El Sistema de SUGERENCIAS}

No pretendemos reducir las posibles vías de participación del personal en la mejora de la calidad de las empresas a las que se analizan y defienden en este artículo, pero sí

2. Argyris, Ch. (1979), El individuo dentro de la organización. Barcelona. Herder.

3. Ouchi, W. (1984), Teoría Z. Barcelona. Ed. Orbis. Martínez Mut, B. (1986), «La teoría Z y las instituciones educativas en la pedagogía de la intervención»en Conceptos y Propuestas (III). Papers d'Educació. Valencia. Nau Llibres, pp. 73-100.

4. Peter, T. y Waterman, R. H. (1983), En busca de la excelencia. Barcelona. Plaza y Janés. Archier, G. y Serieyx, H. (1985), La empresa del tercer tipo. Barcelona. Planeta.

5. López Barajas, E.; Marín Ibáñez, R. y Pérez Serrano, G. (1992), «La investigación-acción», ponencia al XI Seminario Interuniversitario de Teoría de la Educación. Salamanca, p. 10 del documento policopiado. 
conviene especificar que la experiencia personal, así como los datos que justifican las conclusiones que se presentan, se centran de forma significativa en tres tipos de procedimientos: formación y mentalización de los dirigentes (TOTAL QUALITY MANAGEMENT) desde una perspectiva de la calidad total en el seno de las organizaciones, los Círculos de Calidad y el Sistema de Sugerencias.

El tema del liderazgo ya ha sido analizado en otras ocasiones recientes ${ }^{6}$ y es un complemento substancial para la eficacia de los programas que se analizan en este apartado, además de ser -como se verá en el análisis de los datos que se aportan- la base de un grupo de propuestas que para la mejora de los programas presentan los participantes en ellos.

Los programas de participación del personal no surgen como experiencias o acciones aisladas en la gestión de las empresas, sino que se integran dentro de un proyecto más general de cambio o de mejora de la organización, que es, a su vez, el humus que condiciona, pero facilita, la aparición y mantenimiento de aquellos programas.

Quizá sea conveniente, para entender la dinámica y la realidad de esta participación así como las dificultades que surgen en su implantación, considerar las características de estos proyectos de cambio a los que las organizaciones hacen frente en un momento determinado de su evolución o de su existencia. Seis rasgos señalamos a estos procesos:

« $1^{\circ}$. Están provocados generalmente por presiones externas a la organización.

$2^{\circ}$. Los dirigentes tienen conciencia de su necesidad pero no de su alcance ni de las dificultades que puede suponer.

$3^{\circ}$. Es frecuente recurrir al asesoramiento de expertos o consultores externos, pues los dirigentes se sienten más urgidos por responder y gestionar el día a día de la organización.

$4^{\circ}$. Se constata una cierta tendencia de los altos directivos a quedarse al margen, al menos en las fases iniciales del proyecto, del proceso de cambio.

$5^{\circ}$. Suele manifestarse una cierta urgencia respecto a los resultados del proceso, lo que en muchas ocasiones produce el abandono o el fracaso de iniciativas muy loables pero poco sensatas.

$6^{\circ}$. Por último, por parte del personal no existen resistencias al cambio si se explican con claridad los motivos y los objetivos del proceso de cambio, perciben que hay una voluntad real en la empresa de efectuarlo y ven viable y positivo el compromiso que se les pide» ${ }^{7}$.

Por otra parte, el segundo referente que debemos considerar es la existencia de un modelo de programa de intervención socio-educativa, diseñado específicamente para producir el cambio y que se considera adecuado para implantar los programas de participación. Por ello, la evaluación de los resultados y su aplicación a la mejora del propio diseño se apoya en la evaluación de programas o investigación evaluativa, como línea preferente, además de la investigación-acción, para el tema que nos ocupa ${ }^{8}$.

6. Martínez Mut, B. (1991), «Condicionamientos culturales y de liderazgo de los programas de acción socioeducativa» en PAD'E. Vol. 1, n 2, pp. 135-158.

7. «El reciclaje formativo en el mundo laboral: concepto y adaptación a las nuevas exigencias» en Núñez Cubero, L. (ed): Educación y Trabajo. Sevilla, Preu-Spínola, pp. 113-149.

8. Sarramona, J.; Vázquez Gómez, G. y Ucar, X. (1991), «La evaluación de la educación no formal», X Seminario Interuniversitario de Teoría de la Educación. Llanes (Asturias). Documento policopiado de 45 pp. y un anexo. 
De todas formas, conviene insistir en que lo que se pretende es mejorar la práctica educativa o de cambio de la realidad teniendo como marco de referencia un patrón definido como deseable por la empresa implicada en el proyecto, es decir, en este caso una cultura de participación y compromiso con un proyecto o ambición común.

El modelo de programa que defendemos y ha sido utilizado para implantar los Círculos de Calidad y el Sistema de Sugerencias se descompone en las fases siguientes:

1. ${ }^{\mathrm{a}}$ Compromiso inicial de la gerencia de la empresa.

2. Diagnóstico de las actitudes y expectativas del personal respecto a su participación en el programa.

3. Convocatoria y presentación del programa a los participantes potenciales, es decir, los empleados de la empresa.

4. ${ }^{\text {a }}$ Elección de un Equipo de Gestión del programa, que puede o no ser el mismo para ambas vías de participación.

5. Diseño y ejecución de un plan de formación en las técnicas y herramientas de mejora de la calidad propias de estos equipos, tanto para los participantes como para sus líderes.

6. ${ }^{\mathrm{a}} \quad$ Diseño y ejecución de un programa de formación específico para el facilitador y los líderes en temas de conducción de grupos, relaciones interpersonales y motivación.

7. ${ }^{a} \quad$ Funcionamiento tutelado del programa durante un corto período de tiempo.

8. ${ }^{\text {a }}$ Presentación a la gerencia de la empresa de los resultados obtenidos en la fase tutelada.

9. ${ }^{\text {a }}$ Funcionamiento autónomo de los programas.

$10 .^{a} \quad$ Seguimiento y evaluación final.

Levionnois, por su parte, ${ }^{9}$ considera que son necesarias seis etapas para el éxito en la implantación de un programa de Círculos de Calidad e Innovación:

1. ${ }^{a}$ Definir el marco de acción de los CCI.

2. ${ }^{a} \quad$ Informar y sensibilizar al conjunto del personal.

3. ${ }^{a}$ Recogida de las candidaturas y selección de los futuros animadores de los CCI.

4. ${ }^{\text {a }}$ Desarrollo de los ciclos de formación en la dirección (animación y gestión o facilitación y conducción) de los CCI.

5. Puesta en marcha y funcionamiento de los CCI.

6. ${ }^{a} \quad$ Balance de la experiencia: preparación y presentación. Conviene recordar, como otra experiencia, que RENFE denomina a estos equipos Grupos de Iniciativa y Mejora ${ }^{10}$.

\subsection{Los Círculos de Calidad}

Son una vía de carácter colectivo para lograr la implicación del personal en la mejora de la calidad de las empresas a través de la transformación positiva de aquellas tareas que conocen y realizan habitualmente, es decir, su propio trabajo.

9. Levionnois, M. (1992), Marketing interno y Gestión de los Recursos Humanos. Madrid. Díaz de Santos SA., pp. 100-110.

10. Escuela de formación de mandos intermedios (1981), Grupos de Iniciativa y Mejora. Madrid. Publicaciones de RENFE. 
Podemos definirlos de acuerdo con Barra como «un grupo de 4 a 10 empleados que realizan tareas similares y que voluntariamente se reúnen con regularidad, dentro de sus horas de trabajo (también suelen hacerlo en ocasiones fuera de ellas o en horas compartidas), para identificar los problemas de su trabajo, analizar las causas que los provocan y proponer las soluciones adecuadas a la gerencia» ${ }^{11}$. Dentro de esta estructura creada por la empresa, que funciona realmente como uno de sus subsistemas, se consideran necesarias las siguientes figuras o papeles representados por los diferentes participantes en el programa:

- El facilitador o coordinador del programa.

- Los líderes de los Círculos.

- Los miembros de los Círculos,

para cada uno de los cuales se diseñan y ejecutan programas específicos de formación, tal como se ha indicado al analizar las fases en que se descompone el proceso de implantación.

El facilitador o coordinador es la figura que garantiza el contacto del programa de Círculos de Calidad con el exterior y representa la voluntad de la gerencia de comprometerse en el éxito del programa, es decir, en la aplicación de aquellas propuestas que realmente resuelvan los problemas; se encarga de facilitar y promover el trabajo de los diferentes Círculos. La gerencia, por su parte, unas veces directamente y otras a través del facilitador, concreta el compromiso de la empresa a través de tres funciones: autorizar la aplicación de las propuestas evaluadas como factibles y convenientes, explicar las razones por las que algunas de ellas se rechazan y reforzar y recompensar a los Círculos por sus aportaciones.

Los líderes de cada Círculo en ocasiones son elegidos por sus compañeros de grupo después de su constitución formal, en otras la empresa decide que sean los mandos intermedios los responsables de los Círculos, al menos en una primera fase, y es posible también que los propios miembros prefieran que sean sus supervisores quienes conduzcan las sesiones de trabajo. Quizá un diagrama de flujo aclare la estructura y funcionamiento del programa de Círculos de Calidad:

11. Barra, R. (1985), Círculos de Calidad en operación. México, McGraw Hill, p. 57. 

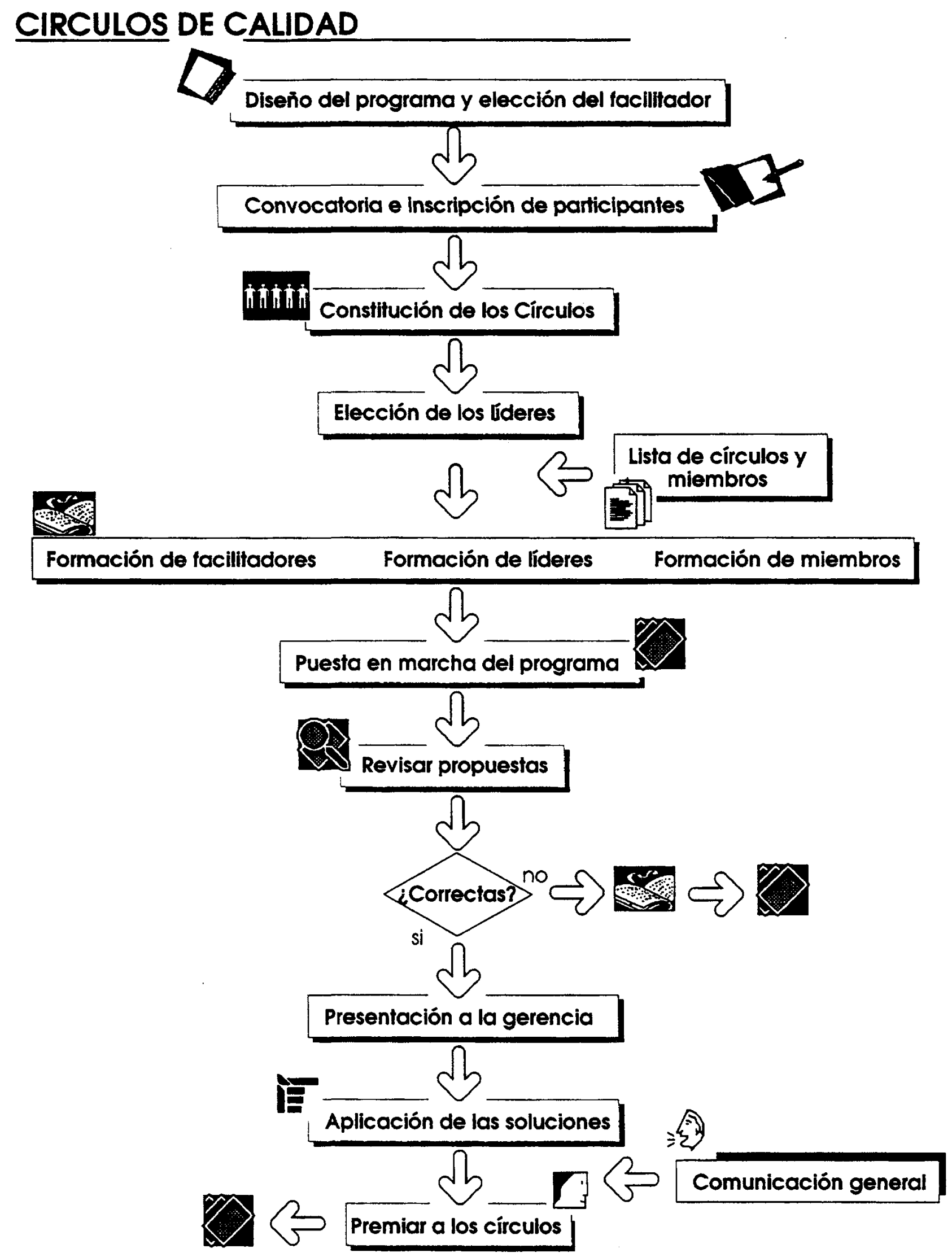
Si analizamos este diagrama de flujo, podremos comprender en cuáles de sus pasos pueden presentarse las dificultades o resistencias a la implantación de las propuestas, derivadas de la participación de los círculos en la mejora de la calidad del trabajo de sus miembros.

En los pasos primero (diseño del programa y elección del facilitador), cuarto (elección de los líderes de los Círculos), sexto (puesta en marcha de los Círculos), séptimo (revisar las propuestas), noveno (aceptación por la gerencia) y décimo (premiar a los Círculos por sus aportaciones) aparecen esos fenómenos, pues es en esos momentos donde pueden manifestarse, y de hecho así ocurre, los intereses contrapuestos de los diferentes departamentos, niveles o áreas de la organización que pueden verse afectados por el programa de los Círculos o implicados en la aplicación de las soluciones que la gerencia autorice.

Por citar algún ejemplo de estas dificultades a las que nos estamos refiriendo:

$1^{\circ} \quad$ Piénsese en cuál será la actitud de un ingeniero del departamento de «ingeniería de procesos» que deba estudiar la viabilidad de una propuesta de mejora en una función o tarea que a él o a su Departamento no se le haya ocurrido, pero sí a un trabajador que en ocasiones apenas sabe representar gráficamente sus ideas y mucho menos defenderlas ante sus jefes.

$2^{\circ}$ ¿Qué postura mantendrá el responsable de controlar los gastos de un Departamento cuando vea que una solución supone un gasto no contemplado en el presupuesto económico del período de tiempo vigente?

$3^{\circ}$ ¿Qué pensará un mando intermedio que vea que sus trabajadores no le eligen como líder del Círculo si lo atribuye a un rechazo a su persona y no a un criterio de representatividad directa establecido en el programa pero que él no comparte?

$4^{\circ} \quad$ ¿Cuál será el comportamiento en la etapa o fase siguiente del programa de un Círculo cuyos miembros vean que sus propuestas se rechazan sistemáticamente después de su presentación a la gerencia?

Además de estos problemas, en el programa concreto de cada empresa las resistencias adoptarán manifestaciones diferentes, pero siempre son muestras de que las personas afectadas ven peligrar aspectos tan importantes para su vida en la organización como el poder, el estatus, etc., por lo que sus resistencias, si no les convencemos de manera preventiva a su debido tiempo, serán fenómenos naturales en el desarrollo de los programas de cambio y serán tanto más fuertes cuanto más importante perciban que es el cambio para ellos.

\subsection{El Sistema de Sugerencias}

Es una vía de carácter individual, generalmente anónima, que pretende estimular el aporte de ideas de aquellas personas que no se sienten inclinadas a participar en un sistema, como el de los Círculos de Calidad, pero que desean contribuir a la mejora de la calidad de la empresa de forma menos estructurada y sin una repercusión conocida e identificada de sus aportaciones.

Un Sistema de Sugerencias, sencillo y propio para pequeñas y medianas empresas, consta de unos elementos personales, individuales y grupales, y otros de carácter físico que actúan como soportes o canales para la comunicación dentro del sistema. 
Los elementos personales son «los miembros de la organización», que pueden enviar sugerencias, y «un equipo de gestión del sistema», encargado de dirigir y mantener en funcionamiento el flujo de comunicaciones, así como de relacionar al sistema con todos los Departamentos de la empresa, y compuesto por personal de los diferentes niveles de la organización. Sus elementos físicos son «la hoja de sugerencias», «el buzón» y el «tablón de anuncios».

Unos elementos funcionales importantes son el sistema de interrelación empresa-sugerencias, así como la forma de recompensar o reforzar aquellas sugerencias que aporten beneficios reales a la organización.

En la aplicación de un Sistema de Sugerencias podemos seguir los siguientes pasos:

$1^{\circ}$ Explicar a los miembros de la empresa o del área afectada los objetivos y razones del programa y lo que se espera de ellos como aportación.

$2^{\circ}$ Definir las funciones del Equipo de Gestión del Sistema, que son:

a) Elaborar las normas de funcionamiento y decidir los elementos físicos que servirán de soporte a la comunicación dentro del sistema.

b) Comunicar a los empleados el inicio del funcionamiento del programa y las demás incidencias que se presenten a lo largo de su desarrollo.

c) Orientar a sus compañeros en la redacción y presentación de las sugerencias.

d) Recoger y evaluar las sugerencias depositadas en el buzón y mejorar aquéllas que lo necesiten aplicando las herramientas de mejora de la calidad.

e) Informar a sus compañeros de la marcha del sistema y de las medidas que se tomen con las sugerencias presentadas.

f) Preparar programas especiales de sugerencias en momentos y para temas determinados.

g) Fomentar entre el personal actitudes positivas hacia la participación y la comunicación, independientemente de su nivel jerárquico.

h) Gestionar el sistema de sugerencias y conseguir el apoyo de la gerencia para la implantación de las sugerencias que presente.

$3^{\circ}$ Decidir los soportes físicos y la estrategia del sistema y comunicarlo a los empleados.

$4^{\circ}$ Determinar los plazos de comunicación de las medidas que se tomen con las sugerencias aprobadas y de las razones por las que otras se rechazan.

5. Elaborar un folleto que explique el sistema de sugerencias y entregar un ejemplar a cada empleado.

6. Instalar los buzones y el tablón de anuncios y poner las hojas de sugerencias al alcance de los participantes.

7. Comunicar el inicio del Sistema de Sugerencias.

Podemos diseñar un diagrama de flujo que explique cómo funciona el sistema: 


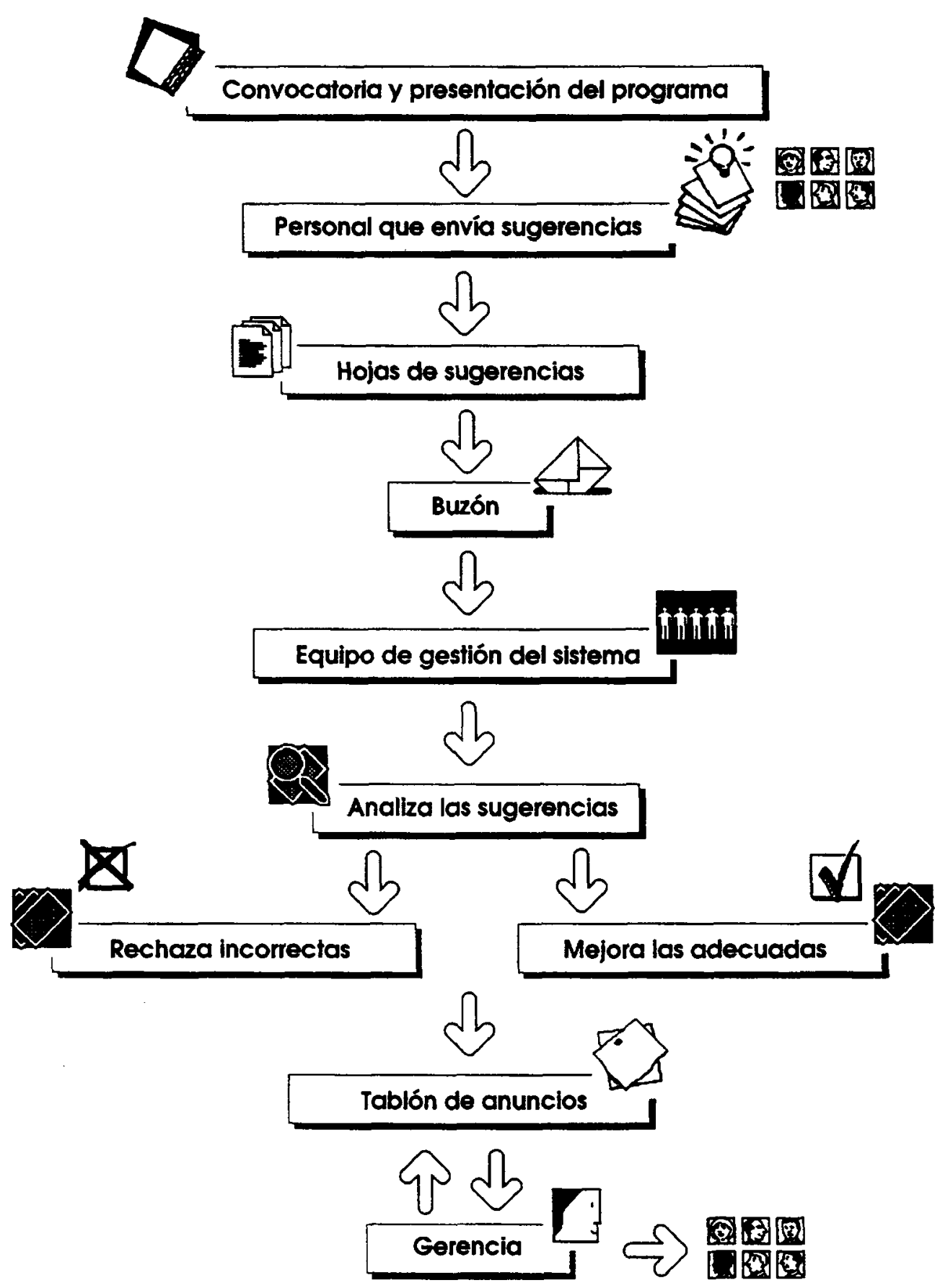

Como hicimos en el diagrama de los Círculos de Calidad, aquí podemos fácilmente descubrir en qué elementos del sistema pueden aparecer las resistencias al cambio o las dificultades para alimentar el flujo de las sugerencias. Nos centraremos en analizar las siguientes:

$1^{\text {a }}$ Que los empleados no envíen sugerencias o envíen solamente quejas o reclamaciones. Aquí definimos la queja como «la manifestación de un estado individual o colectivo de insatisfacción ante la forma habitual de funcionar las cosas en la empresa en general o en algunos de sus departamentos en particular». No llega a aportar ninguna pista de soluciones al problema, pero es una señal de la falta de motivación en el personal que no conviene desatender, pues es origen de problemas latentes, difíciles de objetivar y, por ello, de resolver, pero que, si son bien atendidas, resultan ocasión de propuestas de mejora. 
Su emisor es normalmente la persona que padece la situación deficiente o insatisfactoria o algún otro que se atreve a plantear de hecho esa manifestación.

La causa puede ser un problema real, una situación confusa o mal diagnosticada, también simplemente una forma de protestar del sistema imperante en el ámbito profesional o personal del emisor o de los emisores de la queja.

No es fácil recomendar un camino específico porque cada queja es síntoma de un problema único que, por ello, es difícil de catalogar. Carece de sentido olvidarlas o considerarlas un síntoma indeseable, manifestación del mal funcionamiento de las sugerencias. Debe ser atendida y contestada con la misma seriedad que se utiliza para el Sistema de Sugerencias. Podemos cometer el error de defendernos de ellas y atribuirles una intención de bloqueo del sistema de participación.

$2^{\mathrm{a}}$ Que el Equipo de Gestión realice sus funciones al margen de los Departamentos afectados por las sugerencias, tratando de arrebatarles sus funciones directivas y de toma de decisiones, comunicándose con la Gerencia sin tener en cuenta los Jefes o responsables de aquéllos. Consideramos oportuno e interesante describir la reacción de dos directivos de una empresa en la que se decidió, de acuerdo con el Director Gerente y después de lograr el apoyo de todos los directivos, implantar un programa de Círculos de Calidad y un Sistema de Sugerencias: «un grupo de supervisores de la empresa X (está entre las que se citan en las referencias bibliográficas y fuentes) se ha comprometido junto con su director gerente en el diseño y aplicación de un programa de mejora de la gestión, en cuyas tareas se ha implicado desde el principio este grupo de personas, colaboradores y subordinados directos del director gerente. Como consecuencia de ese programa se acepta la puesta en marcha en todos los Departamentos de la empresa de dos proyectos sencillos de participación del personal en la mejora de la empresa: los Círculos de Calidad y el Sistema de Sugerencias.

De dos de los Departamentos no se inscribe para formar parte de los Círculos ningún empleado, decidiéndose entonces aplicarlo como piloto en uno de los Departamentos, cuyo supervisor dirige el equipo de gestión del proyecto. En la primera fase del proyecto de sugerencias, de esos dos Departamentos tampoco surgen aportaciones y ambos directivos, en las reuniones de supervisión y seguimiento de los proyectos, no plantean dificultad ni resistencia alguna a su continuidad. Cuando en la segunda fase sus subordinados aportan sugerencias que les afectan y tienen que comprometerse en aplicar aquéllas que el Equipo de Gestión, como ocurría hasta entonces, considera viables y adecuadas, tratan de descubrir quiénes son los que las envían, pese a que son anónimas, menosprecian las aportaciones y tratan de boicotearlas por impertinentes cuando analizan las que no pueden rechazar.

En la siguiente reunión de seguimiento de ambos proyectos, en presencia del Director Gerente de la empresa, afirman que los proyectos son inútiles, suponen gastos y exigen esfuerzos suplementarios para lograr que los empleados participen y, cuando lo hacen, no hay garantías de que sus aportaciones resuelvan los problemas importantes».

$3^{\text {o }}$ Que la Gerencia no aplique las sugerencias o no explique las razones por las cuáles no se consideran aplicables algunas de las aportadas.

\section{ANÁLISIS DE LOS DATOS OBTENIDOS EN LAS EMPRESAS}

Los datos que se presentan en este apartado son las contestaciones de casi un centenar de participantes de siete empresas de la Comunidad Valenciana, cinco de Valencia, 
una de Castellón y otra de Alicante $^{12}$, todas ellas comprometidas en algún tiempo con proyectos de mejora de la calidad, dentro de los cuales se han formulado y aplicado los programas de participación objeto del presente artículo comunicación. Durante los meses de enero y febrero de 1993 he asesorado un nuevo programa de participación a través del Sistema de Sugerencias en el Centro El Azud de Tuéjar (Valencia) y se puede afirmar que los datos obtenidos al diagnosticar las actitudes de los participantes no alteran significativamente los que se obtuvieran en las siete empresa iniciales.

El procedimiento para la obtención de los datos ha sido en todos los casos una sesión especial utilizando la técnica METAPLAN ${ }^{13}$, que fundamentalmente consiste en repartir doce cartulinas a cada participante para que, en cada grupo de cuatro, exprese sus ideas respecto a:

$1^{\circ}$ situación ideal o modelo de organización;

$2^{\circ}$ dificultades para conseguir una participación real en la mejora de la empresa, y

$3^{\circ}$ propuestas que formulan para acercarse a ese modelo ideal.

Cada participante rellenó en silencio y de forma individual y anónima sus cartulinas para evitar las influencias de los demás a la hora de anotar sus ideas. Posteriormente cada uno podía seleccionar las propias o las de los demás también de manera anónima y silenciosa.

En muchas sesiones de trabajo en grupo se manifiestan fenómenos de dinámica interna y externa que puede actuar como frenos o bloqueos a la participación de todos sus componentes y a la libre circulación de las ideas que son patrimonio del grupo. Estas «transacciones ulteriores», si utilizamos la terminología del Análisis Transaccional ${ }^{14}$, provocan que los receptores de los mensajes confusos, por competencia entre ellos o entre sus canales, atiendan de forma preferente al mensaje no verbal, implícito u oculto, con lo que la comunicación se desvía de su propósito original. Precisamente una de las formas de evitar estas presiones o bloqueos es utilizar una herramienta de pensamiento divergente como es el Metaplán.

Así ocurre que los miembros del grupo, el cual es dueño de las ideas una vez se presentan en las cartulinas, atribuyen puntos a ideas de otros y no les produce valoración negativa alguna dejar de puntuar las que cada uno aportó en principio.

Las respuestas obtenidas, como resultado de la aplicación de esta herramienta de trabajo grupal, se han porcentualizado para normalizarlas, pues en todos los casos las cartulinas no tenían el mismo número de ideas ni cada grupo recibía el mismo número de respuestas. Todas las ideas han sido consideradas, aunque algunas solamente hayan obtenido una sola frecuencia de aparición.

12. Archivo del Departamento de Teoría de la Educación de la Universidad de Valencia y Archivos de la Empresa GCC S.A. (Intur, Walon Ibérica, AIADHESA, Ibérica de Aparellajes, SOGEVA, SEVAL, IMPIVA y CENTRO EL AZUD.

13. Metaplán, «Instrucciones para su aplicación». Documento de trabajo del autor, policopiado.

14. Kertesz, R.; Atalaya, C. I.; Kertesz, V. R. (1992), Liderazgo Transaccional. Buenos Aires. I.P.P.E.M., pp. 21 y ss. Se denominan en el Análisis Transaccional «transacciones ulteriores» aquellas que activan a la vez varios estados del yo en el emisor y/o en el receptor». 


\subsection{Situación ideal o modelo de empresa}

Aparecen un conjunto de valores que se pueden agrupar en dos subconjuntos:

$1^{\circ}$ Referidos a la empresa en su conjunto. Estructura y funciones (36.8\%): Información y comunicación; libertad, iniciativa y autonomía; claridad de objetivos; unión y cohesión; medios técnicos y personales, y formación; Relaciones Humanas (15.6\%): Reconocimiento, respeto, consideración, relaciones personales, buen trato a los clientes, valoración económica, ambiente laboral agradable y promoción; Éticos (8.9\%): Confianza y lealtad, ética (responsabilidad y consecuencia), sinceridad y seriedad.

$2^{\circ} \quad$ Referidos a rasgos o características de los dirigentes. Profesionalidad (26.7\%): Emprendedor e innovador, eficaz y orientado a las tareas, enérgico (con empeño y audacia), inteligente o estratega, flexible, coordinador, realista y objetivo y con memoria; $A c$ titudes hacia sus subordinados (7.4\%): Abierto al diálogo, democrático, comprensivo; Rasgos éticos (4.4\%): carisma, visión y fe, identificación con el proyecto, responsable y consecuente.

\subsection{Problemas reales o dificultades actuales}

Impiden o dificultan la aplicación de lo que se plantea como modelo ideal. Por orden de frecuencia han sido los siguientes: Falta de motivación (14.5\%), carencias de información (13.1\%), presión en el trabajo y escasez de tiempo para reflexionar $(11.1 \%)$, falta de claridad en metas y objetivos $(9.7 \%)$, falta de medios técnicos y humanos $(9.7 \%)$, incomprensión y falta de diálogo $(8.7 \%)$, carencias de formación $(8.2 \%)$, burocracia y papeleo $(6.7 \%)$, sistema de formación poco práctico y no adaptado a las necesidades de la empresa (5.3\%), autoritarismo y poca autonomía (2.4\%), mi jefe directo (1.9\%), falta de colaboración y confianza entre jefes y subordinados $(1.9 \%)$, falta de seguimiento y continuidad de los programas (1.9\%), inseguridad en el trabajo (1.9\%), falta de unión entre todos $(0.9 \%)$, problemas económicos $(0.9 \%)$, dificultades para cambiar la mentalidad de las personas $(0.5 \%)$.

\subsection{Propuestas de mejora}

En este grupo se presentaron el mayor número de ideas de los participantes. Sus agrupaciones quedan así:

$1^{\mathrm{a}}$ Relativas a las acciones de formación (24.2\%): mayor profundidad a los temas, explicar su trascendencia para todos, conocer la situación real de la empresa, utilizar ejemplos prácticos y reales, participación activa en la preparación de los cursos, seleccionar previamente a los participantes realmente interesados, acortar tiempo y concentrar los temas, asistencia obligatoria, no tener trabajos pendientes, dentro del horario laboral, fuera del lugar del trabajo, con MAV y adaptaciones a la vida real, formación sobre temas del oficio, idiomas y que sea continuada dentro de un plan.

$2^{\mathrm{a}}$ Referidas a la organización (22.5\%): definir objetivos comunes, eliminar compartimentos estancos, definir criterios de actuación, seleccionar prioridades, definir responsabilidades y funciones, optimizar los recursos, mantener relaciones con el exterior, 
reestructurar las plantillas, elaborar manuales de organización, facilitar el cambio por la propia empresa, tener capacidad de respuesta, mejorar el organigrama, gestión eficaz y transparente, seguimiento del funcionamiento, cuidar el rigor y el detalle, adaptarse a las directrices de los órganos directivos.

$3^{\mathrm{a}} \quad$ Referidas a la mejora de la comunicación (15.1\%): importancia de la información hacia el cliente, avisos a tiempo, información frecuente sobre logros, igual información para todos, medios claros según los usuarios, relaciones entre la dirección y los departamentos, canales formales adecuados, sistema de sugerencias, explicación de las respuestas negativas.

$4^{\mathrm{a}} \quad$ Relativas a la motivación del personal (6.1\%): trato personal exquisito, implicar a todos, valorar resultados, incentivos materiales, autoconvencimiento, actitudes positivas, no crear falsas expectativas, espíritu de trabajo.

$5^{\mathrm{a}}$ Necesidad de un liderazgo compartido basado en las siguientes funciones o rasgos $(5.7 \%)$ : coordinación, confianza, colaboración, visión global de la empresa, coherencia, unificación de criterios, dar ejemplo.

$6^{\mathrm{a}}$ Elaborar y aplicar un plan sistemático de reuniones (5.5\%): «formar grupos», colaboración y compañerismo, libertad de reunión, trabajo en equipo, contactos entre directivos.

$7^{\mathrm{a}}$ Mejorar las relaciones humanas (4\%): respeto, actitudes positivas hacia los otros, clima laboral agradable, actitud de respeto hacia los subordinados.

$8^{\mathrm{a}} \quad$ Mejorar de los medios, instalaciones, métodos y logística (3.8\%).

$9^{\mathrm{a}}$ Los programas que sean respuesta a las necesidades reales de la industria $(3.7 \%)$.

$10^{\mathrm{a}}$ Dotar de mayor nivel de responsabilidad y autonomía en las decisiones (3.7\%): participar en la fijación de objetivos, participar en la toma de decisiones, asumir responsabilidades.

$11^{\mathrm{a}}$ Fomentar la conciencia e imagen de la calidad de servicio como cultura de la empresa (2.9\%).

$12^{\mathrm{a}}$ Otras: apoyo de la dirección (1.1\%), menor presión de trabajo y más tiempo libre para reflexionar $(0.9 \%)$, trabajar todos con honradez $(0.2 \%)$, estudiar nuevos mercados $(0.2 \%)$.

\section{CONCLUSIONES Y PROPUESTAS DE MEJORA}

Hay que decir que los doce grupos de propuestas, realizadas por estos anónimos participantes en los programas de mejora, son asumibles en su totalidad, puesto que surgen de las dificultades que han sentido para aplicar lo que idealmente se les había propuesto o aquello de lo cual se habían formado las correspondientes expectativas.

Poniendo en relación estas ideas con el proceso o programa de intervención que se ha presentado en el apartado segundo, se concluye que habrá que reforzar o dar mayor relevancia a las fases siguientes:

$1^{\text {a }}$ Compromiso inicial de la Gerencia de la empresa: «insistir en la necesidad de un compromiso real en la aplicación de las propuestas factibles».

$3^{a}$ Convocatoria de inscripción y presentación del programa a los participantes: «no crearles falsas expectativas que luego no se vayan a resolver y explicar con claridad la conexión entre los programas de participación y el proyecto general de mejora». 
$5^{\mathrm{a}}$ Diseño y ejecución de un plan de formación en las técnicas y herramientas de trabajo propias de estos equipos «insistiendo, en el caso de sus líderes, en las funciones de facilitación y refuerzo para que no decaigan el interés y los deseos de participación» ${ }^{15}$, y, «en el caso de los programas, que sean lo más específicos posible para las necesidades de los grupos en el seno de la empresa». Una consecuencia de la aplicación conjunta en la misma empresa del Programa de Círculos de Calidad y del Sistema de Sugerencias es la polifuncionalidad del programa de formación en las herramientas de mejora de la calidad, que puede ser impartida tanto a los miembros de los Círculos de Calidad como a los integrantes del Equipo de Gestión del Sistema de Sugerencias, que las necesitará para convertir una simple queja en sugerencia o para mejorar algunas de las que aporten los participantes en el sistema.

$7^{\mathrm{a}}$ Presentación a la Gerencia de los logros obtenidos en esta fase tutelada: «compromiso público de ésta en la aplicación de las medidas o explicación de las que no sean factibles».

$8^{\mathrm{a}}$ Funcionamiento autónomo de los programas: «facilitar su funcionamiento dentro de las condiciones pactadas al principio».

$9^{a}$ Seguimiento y evaluación final: «utilizar indicadores objetivos previamente seleccionados y cuantificados».

Habría que añadir una fase antes del inicio del funcionamiento autónomo del programa: «creación de un sistema claro, objetivo y fiable de comunicación y de reconocimiento de las aportaciones de los equipos y de los empleados individuales, en el que la ida y el retorno de la información fluyan de forma rápida y eficaz en todos los sentidos».

Una reciente innovación en la búsqueda de mejores sistemas de implicación del personal en la mejora de la calidad son los Equipos Integrados de Mejora, constituidos unas veces como estructuras «ad hoc» para el logro de determinados objetivos específicos (la empresa Continente, S. A. los implanta para mejorar la satisfacción de sus clientes por el servicio recibido), y otras, como procedimientos ordinarios para el desempeño en grupo de las funciones de los puestos de Trabajo (Ford España, S.A. los denomina Manufacturing Integreated Teams o Equipos Integrados de Mejora y los está implantando en su fábrica de motores de cara a la fabricación del motor Sigma).

\section{BIBLIOGRAFÍA}

ARCHIER, G. y SERIEYX, H. (1985). La empresa del tercer tipo. Barcelona. Planeta.

ARCHIVOS DEL DEPARTAMENTO DE TEORÍA DE LA EDUCACIÓN DE LA UNIVERSIDAD DE VALENCIA Y ARCHIVOS DE LA EMPRESA GCC, S.A. (Intur, Walon Ibérica, AIADHESA, Ibérica de Aparallajes, SOGEVA, ASEVAL, IMPIVA y CENTRO EL AZUD).

ARGYRIS, CH. (1979). El individuo dentro de la organización. Barcelona. Herder.

Barra, R. (1985). Círculos de Calidad en operación. México. McGraw Hill.

15. López Mena, L. (1989), Intervención psicológica en la empresa. Barcelona. Martínez Roca, pp. 33-48. 
ESCUELA DE FORMACIÓN DE MANDOS INTERMEDIOS (1989). Grupos de Iniciativa y Mejora. Publicaciones de RENFE. Madrid.

KeRTesz, R.; Atalaya, C. I. y KeRstesz, V. R. (1992). Liderazgo Transaccional. I.P.P.E.M. Buenos Aires.

LEVIonNoIs, M. (1992): Marketing interno y Gestión de los Recursos Humanos. Madrid. Díaz de Santos, S.A.

LóPez Barajas, E.; MARÍN IbÁÑez y PÉrez Serrano, G. (1992): «La investigación-acción», ponencia al XI Seminario Inter-universitario de Teoría de la Educación. Salamanca.

LóPEZ MENA, L. (1989). Intervención psicológica en la empresa. Martínez Roca. Barcelona.

MCGREGoR, D. (1969). El aspecto humano de la empresa. Ed. Diana. México.

MARTíneZ MuT, B. (1986). «La teoría Z y las instituciones educativas en la pedagogía de la intervención» en Conceptos y Propuestas (III). Papers d'Educació. Nau Llibres. Valencia.

- (1991). «Condicionamientos culturales y de liderazgo de los programas de acción socioeducativa» en PAD'E. vol, $1, n^{\circ} 2$.

- «El reciclaje formativo en el mundo laboral: concepto y adaptación a las nuevas exigencias» en NúÑEZ CUBERo, L. (Ed.). Educación y Trabajo. PREU-Spínola. Sevilla.

METAPLÁN. «Instrucciones para su aplicación». Documento de trabajo del autor, policopiado.

OUCHI, W. (1984). Teoría Z. Ed. Orbis. Barcelona.

Peter, T. y WATterman, R. H. (1983). En busca de la excelencia. Plaza y Janés. Barcelona.

SARRAMONA, J.; VÁzQUEZ GÓmEZ, G. y UCAR, X. (1991). «La evaluación de la educación no formal», X Seminario Interuniversitario de Teoría de la Educación. Llanes (Asturias). 